\title{
Emergence of the Collective Oscillations in Electron Energy Loss Spectra of $d$ - electrons in III-V Nitrides
}

\author{
Rohan Dhall ${ }^{1}$, Houston Dycus ${ }^{1}$, Derek Vigil Fowler ${ }^{2}$ and James M. LeBeau ${ }^{1}$ \\ 1. Department of Material Science and Engineering, North Carolina State University, Raleigh, NC, USA \\ 2. Material Science Center, National Renewable Energy Laboratory, Golden, CO, USA
}

In this work, we study the electron energy loss spectra of Aluminum-Gallium Nitride (AlGaN) materials. In particular, we focus on the low loss region of the EEL spectra, which show deviations from the classical Drude picture due to solid state effects. A comparison with ab-initio calculations yields insight into the origin of specific features in the observed spectra, and the role of electron-plasmon interaction.

We observe a dominant bulk plasmon, which originates from the collective oscillation of valence electrons in the $\mathrm{AlGaN}$ system. As the Gallium fraction is increased, this bulk plasmon shifts and broadens. These shifts can be explained by a simple Drude model, in which the resonant energy of plasmon oscillation is related to the charge density of valence electrons in the system. However, this description is not sufficient to explain the emergence of additional peaks in the recorded EEL spectra.

An alternate picture is proposed, in which, not all valence electrons in the system are treated as identical. Instead, the wavefunctions of the valence electron states are computed using ab-inito methods in a self consistent field approximation, and many-body effects are included using the $G W$ approximation. The polarizability (and hence, the dielctric function) of the nitride materials is calculated within this approximation, and the resultant loss function does indeed show the emergence of additional features with increasing Gallium fraction.

We then systematically eliminate specific states from the calculation of polarizability and the dielectric function in Gallium Nitride. We find that when the Gallium $d$-electrons are removed, from this summation, the additional peaks observed in the loss function disappear. This leads to the conclusion that these features in the EEL spectra arise from the polarization and oscillation of the $d$-electrons contributed by Gallium atoms.

Similar deviations from the classical Drude picture have been observed before in other material systems. For instance, graphitic carbon has distinct plasmon modes, which arise from the oscillation of $\mathrm{s}$ and $\mathrm{p}$ electrons[1]. Similarly, it has been reported that coupling between electrons and plasmons can significantly alter the lifetimes of the plasmon, and modify the resultant EEL spectra. Due to their large bandgaps, nitrides provide a material system where such a coupling is expected to play an important role in accurately describing the EEL spectra of the material. 


\section{References:}

[1] Lischner, Johannes, Derek Vigil-Fowler, and Steven G. Louie. "Physical Origin of Satellites in Photoemission of Doped Graphene: An Ab Initio G W Plus Cumulant Study." Physical review letters 110.14 (2013), p. 146801.
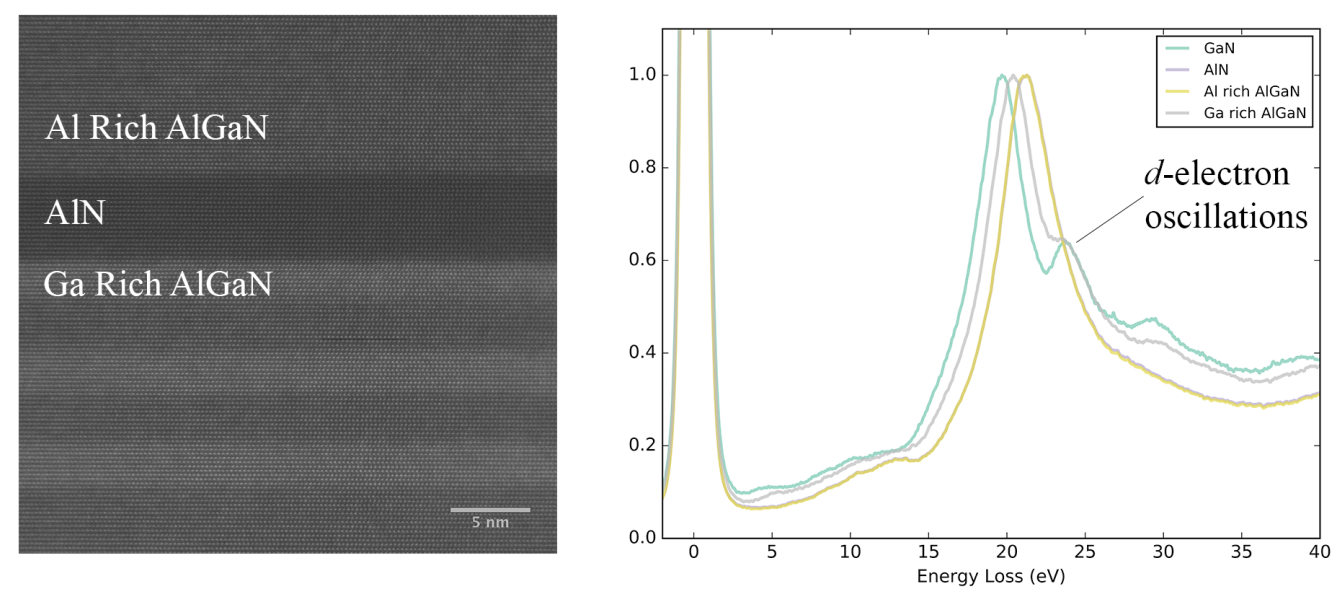

Figure 1. A HAADF image of AlGaN quantum wells, with the low loss EEL spectra for Gallium and Aluminum rich regions. The shoulder peaks at $23 \mathrm{eV}$ arise as the Gallium fraction increases.

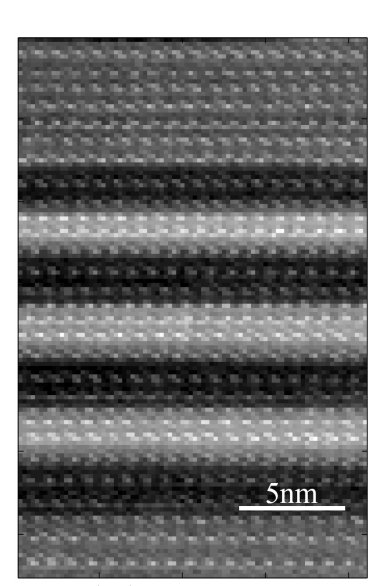

HAADF Image

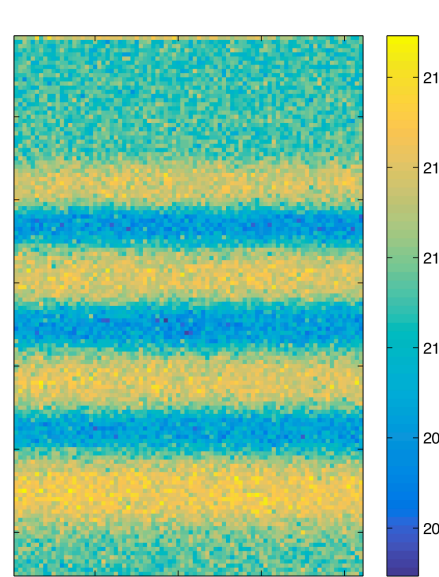

Plasmon Energy $(\mathrm{eV})$

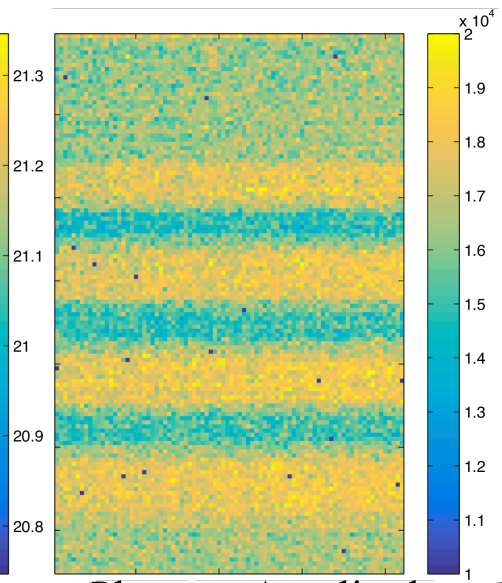

Plasmon Amplitude

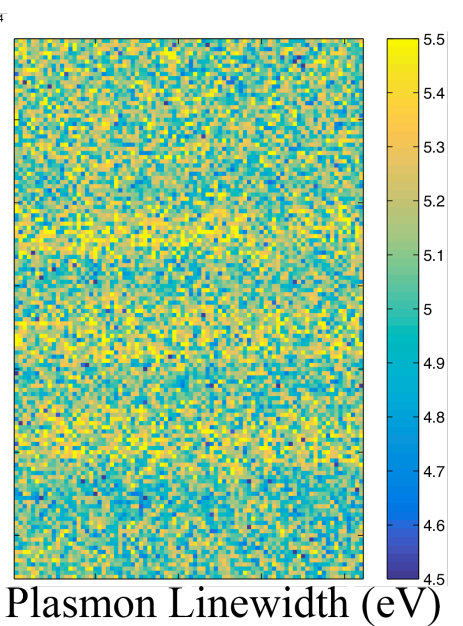

Figure 2. A HAADF image of AlGaN quantum wells, with the Gallium rich regions appearing brighter. The plasmon peak in the low loss EELS is fit to a peak function to generate maps of the plasmon energy, amplitude and linewidth. 